CANADIAN JOURNAL OF PHILOSOPHY

Supplementary Volume 32

\title{
Biological Modules and Emotions
}

\author{
PAUL DUMOUCHEL
}

But as for most genes, they are not the units of interest once we get to the network level: it is the whole conspiracy we care about. (von Dassow and Meir 2003, 27)

\section{Biologists and Modules}

Biologists, more precisely evolutionary biologists, and not only psychologists and philosophers also speak of modularity. However the way in which this theoretical construct functions in their discipline is relatively different from the role it obtains in evolutionary psychology and cognitive science. Rather than postulating modules to explain particular traits of organisms, such as the specificity of input systems or limitations of human reasoning abilities, biologists originally simply assume that some form of modularity constitutes a precondition of evolution. ${ }^{1}$ They argue that, in order for natural selection to fine tune organisms to their environment, different traits must be able to evolve independently from one another. This implies modularity in one form or another. Organisms will only be able to adapt if they do not come all in one piece, so to speak. It must therefore be possible for changes to occur in one characteristic of an animal without those changes having repercussions throughout the whole organism. It seems that evolution requires that organisms be modular; it requires that they be made of relatively independent building blocks that are nonetheless in some way integrated. How is this delicate bal-

1 Sloman (2002) applies a somewhat similar understanding of modules to emotions and the cognitive domain in general. 
ance achieved? Is it possible to identify, describe, and analyze these modules?2

As it turns out, biologists have been quite successful in their search for modules, perhaps too much so. They have come back with many different kinds of modules and more questions than they started out with. Among other things, some types of modules appear to be obstacles to continuing evolution and adaptation rather than necessary conditions for it to take place. Furthermore, it should be added that at this point in biology many questions concerning modules still remain unanswered. Biologists disagree as to how many different types of modules there are; they disagree concerning which conditions and how many conditions something must satisfy in order to qualify as a module; they disagree about the role and importance of modules in evolution; finally, they even sometimes disagree as to whether this or that object is a module or not. What they do agree on is that there are such things as modules in organisms, and they can exhibit a fairly long list of objects that have a good claim to being called modules. By saying "objects that have a good claim to being called modules," I mean to insist upon the fact that, even if biologists resort to definitions and conceptual analysis, they essentially work with biological objects, organisms, processes, and structures. Rather than elaborating an abstract model of what a module is and then seeing which objects qualify to be a module in view of this model, as in a sense Fodor (1983) does, they exhibit exemplars, objects to which they point as examples of biological modules. ${ }^{3}$ Their claims are about these objects. They describe, for example, a genetic pathway, and then argue that there are good evolutionary or developmental reasons to call the object a module. They argue that considering it to be a module makes sense and that it is useful and explanatory.

In what follows, I first want to rapidly review a few examples of biological modules. These modules, or at least a sub-category of them, constitute an interesting class of objects. They are described and ana-

2 Granted, this is a rational or conceptual reconstruction of the search for modules rather than a historically adequate description of the way in which modules became an important topic in evolutionary biology.

3 For a similar understanding of the way biologists work with objects, exemplars, and model organisms rather than abstract models, see Kelley (2002), 51-52. 
Biological Modules and Emotions

lyzed at the sub-cellular level; however, their consequence, what they are taken to explain or to do within a biological organism (or between organisms), is located at the level of a cell population, of a group. In other words, biological modules of the type described below are implemented at the sub-cellular level in the sense that they are contained within the individual cell but their effect, their role, is located at the level of a whole cell population. Emotions, or rather affective coordination, I then wish to argue, with the help of a short fiction, may be viewed as resting on a similar type of sub-personal modules that lead to consequences that are visible at the level of a group or population.

\section{Biological Modules}

Biologists mainly distinguish between evolutionary and developmental modules. In both cases, a module can correspond either to a structure (for example, specific types of cells or an organelle) or to a process. In what follows, I will be mainly interested in modules that are process. Classical examples of this type of module are regulatory genetic networks. Such networks are processes rather than structures inasmuch as they do not exist other than as a collection of chronologically arranged events where the activation of one gene leads, for example, to the activation of another, and the two activated together result in the inhibition of a third, and so on. There is no material structure that corresponds to the network. The network is just the sequence of interrelated events. Genetic regulatory networks qualify as biological modules when they exhibit all or most of the following characteristics.

First, they are relatively autonomous in the sense that, once initiated, the sequence of events follows its course independently of the environment, and in the sense that the network is to some extent selfsustaining. Of course independence from the environment is never absolute and there are changes in the environment that will prevent the network from functioning. Nonetheless, as long as the specific products are present, or certain parameters maintained within a definite range, the chain of events takes place unperturbed. Theoretically the advantages of autonomy are readily evident. It allows the network to be deployed in many different contexts where it can nonetheless perform correctly. Interestingly enough, this is precisely what we find. 
One of these genetic regulatory networks, named the Pax/Six/Eya/Dach network, plays a fundamental role in differentiation of the cells that become photoreceptors in the eye of Drosophilia. Among vertebrates this same network is indispensable for the development of muscles, eyes, and ears (Kardon et al. 2003, 63). Diversity of usage is the second characteristic of biological modules. Modules are used over and over again in many different contexts, as if they were good tricks that, once hit upon, should not be abandoned lightly. This is true of the $\mathrm{Wg}$ signalling pathway which has short- and long-range functions during cell epidermal specification and the core elements of which have been preserved in many species including Drosophila, C. elegans, Xenopus, chicken, mouse, and human (Borycki 2003, 105). It is also true of the Notch signalling module that influences cell fate choices during the development of multicellular organisms. It has essentially been conserved in vertebrates and invertebrates, and it affects similar developmental operations in all organisms where its function has been analyzed (de Celis 2003, 81). As Strähle and Blader say:

[W]e define a module as an assembly of biological structures that fulfill a function in an integrated and context insensitive manner. Function as defined here is not merely the interaction of molecules but an interaction that yields a biological output which is characteristic of the module. Furthermore, the application of the module is flexible. To be recognized as a module, it has to be used either in different processes in the same organism or in different organisms, exploiting its invariant functional properties in the same or different processes. A module is therefore characterized by its reiterated use. (Strähle and Blader 2003, 35)

The third characteristic, as suggested by the previous examples, is that modules are stable over very long periods of time. The same modules are not only employed in different cellular contexts within one organism or species of organism; they are found in many different species that often are phylogenetically quite distant. It should be added that a module as it exists in different contexts or species is not always strictly identical. For example, what we often find in different species is not exactly the same genetic network but a highly similar one. Such is the case of the Pax/Six/Eya/Dach network mentioned earlier. In vertebrates it does not mobilize exactly the same genes as in Drosophilia, but calls 
Biological Modules and Emotions

upon genes that belong to the same family of genes or on homologous genes. This last point is one of the reasons why biologists often disagree about the role of modules in evolution. On one hand the fact that modules evolve, that they are not always the same in different species, suggests that natural selection can fine tune them to different environmental requirements. On the other hand, the phylogenetic stability of modules, the fact that what we are dealing with nonetheless are different versions of the same modules that play similar functional roles in many different cellular contexts and species, suggests that modules constitute strong constraints that limit the paths that are open to evolution.

The fourth characteristic of biological modules is that they often are hierarchically organized in the sense that lower-level modules, i.e., signalling modules, can be part of higher-level modules. For example, the Notch signalling module is a necessary part of the Basic Helix-Loop-Helix protein domain module that plays a pivotal role in neurogenesis in both vertebrates and invertebrates (Strähle and Blader 2003, 39). As a consequence of their relative autonomy, which among other things allows them to be imbedded in higherlevel modules, modules can also "frequently be triggered in a switch like fashion by a variety of inputs ... to which they are only weakly linked ... and may affect different downstream processes depending on the circumstances" (Schlosser 2003, 525). Thus, as José de Celis argues: "Although the Notch signaling pathway is a context independent module, in the sense that the molecular interactions between its members are conserved and invariant, the outcome of Notch signaling is highly context dependant" (de Celis 2003, 93). Anne-Gaelle Borycki reaches a similar conclusion concerning the Sonic Hedgehog and Wnt signalling pathways: "despite their apparent autonomy, genetic modules are subject to and part of larger signaling network, which remodels them into context-dependent modules." (Borycki 2003, 121). It follows from this, as Schlosser clearly noted, that modules can be described as being the same only if they are described in a sufficiently abstract way. For example, two processes in which the Notch signalling network plays a significant role are lateral inhibition and the formation of boundaries in cell populations, but both processes can lead to quite different end results depending on the context (de Celis 2003, 91). "Despite qualitative differences in inputs 
and outputs in these cases," says Schlosser, "the quantitative and spatio-temporal input-output transformation of the module - what could be called its logical role (or its intrinsic behavior ...) - stays the same." (Schlosser 2003, 525). In other words, there must be a description of the module's operation under which it remains the same, but this description will usually be in terms of intra-modular interactions and of input-output relations defined spatio-temporally, e.g., we go from this molecule to that molecule, rather than qualitatively. On the contrary, as far as its qualitative consequences are concerned, the module's result will vary from domain to domain. It is only under a sufficiently abstract description that the module can be described as "doing the same thing."

Finally, and this last point is fundamental, the operation of modules like Sonic Hedgehog and Pax/Six/Eya/Dach yields consequences that exist at a level that is different from the one at which the module is described. What I mean is that to describe a signalling pathway like Sonic Hedgehog, you analyze molecular events that are taking place at a sub-cellular level, but the end result of its functioning, which is often what you are actually trying to explain, or what is defined as (one of) the role(s) of the module, is something that takes place at the level of a cell population, for example, the creation of a border between two cell groups or a pattern of cellular differentiation, neurogenesis, etc. This is particularly clear for signalling modules, but it is also true of positional modules, as well as of the module that is responsible for cellular suicide (Apoptosis) and that has remained to some extent similar from C. elegans to us (Ameisen 1999). Overall, it is true of all the genetic regulatory networks that work together during development and that have been conserved in a wide range of metazoan taxa. There is a sense in which these modules supervene on more than one individual. It is true that they are implemented individualistically in the sense that the elements of the network exist inside one cell and the interactions between these elements are a purely internal process. Nonetheless, biologically, their result does not make sense at the level of a single individual. It does not make sense not only because, biologically, an isolated individual of one particular cell type plays absolutely no role or function within an organism, such an isolated cell is only a meaningless accident, but also because the inappropriate differentiation of a single cell normally rapidly leads to cellular 
Biological Modules and Emotions

suicide. ${ }^{4}$ Furthermore, modules that are found and implemented in unicellular organisms also play the role of coordinating populations of organisms. Such modules typically constitute the basis of self-organizing processes in cell populations (Camazine et al. 2003, 91-115). These biological modules that have been maintained for a long time through a wide range of species are infra individual modules indispensable for the coordination or collective behaviour of populations of individuals. To paraphrase what Hobbes said concerning justice, the characteristic of cells supported by such modules belong to cells in society, not in solitude (Hobbes 1651).

In the remainder of this paper I wish to consider modules in the affective domain in a somewhat similar way to what biologists do. We usually understand the modularity of emotion as meaning that there are modules (in a relaxed Fodorian sense of module) 5 that underlie or constitute certain emotions. One of the problems to which this hypothesis has led is a fractioning of the category of emotions. Some instances of fear, anger, or disgust, for example, seem to correspond pretty well to the functioning of a module, but other instances apparently do not. In order to accommodate these anomalous examples, one popular strategy has been to distinguish between basic emotions which correspond to hardwired modules and higher-level emotions that are more cognitive and depend to some extent, in an unspecified and obscure way, on those modules. In other words, we associate modules with individual emotions, and when that association proves for some reason inadequate, we redraw the borders of the emotion leaving out for future research those instances that do not fit the modular explanation. This may or may not be good science. However the main difficulty as I understand it lies with the association of modules and particular emotions. This association is equivalent to defining the function of affective modules at the individual or personal level, given that emotions, whatever else they may be taken to be, are viewed as individual subjective events. Emotion modules are seen as playing a

4 I say "normally" because one thing that seems necessary for the growth of cancers is that isolated cells fail to commit suicide. Cf. Ameisen (1999), 133-50.

5 See Ronald de Sousa's contribution to this issue concerning the applicability of Fodor's conception of modules to emotions. 
role for individual agents; they produce or realize certain emotions that are beneficial to the individual, or at least that are supposed to have been beneficial during the EEA (Cosmides and Tooby 2000). The idea I want to pursue is that of affective modules that are internal to an individual entity and best described at the infra-individual level, but whose consequence, role, or function is located at the level of the population or at least of coordination between individuals. What I want to focus on are affective coordination and expression. Affective modules in the above sense, if they exist, are relatively autonomous processes whose functioning remains the same in a wide range of environments. It is likely that among these there will be processes that have been around for a long time; processes that we share with some of our distant ancestors. Furthermore, we should also expect such modules to be recruited for more than one purpose or function, to be "characterized by [their] reiterated use" as Strähle and Blader write and sometimes to be hierarchically imbedded in one another. Therefore, if such modules exist, there is no reason to expect that they will map neatly onto our categories of emotions. This is because emotions are defined at the level of the individual and like biological modules that give different results in different context we should expect affective modules to do the same. In other words, affective modules, like biological modules locally at the sub-individual level, can be doing exactly the same thing, while distally, at the population level, they may be doing something entirely different. Yet emotions are defined at neither of these two levels but at the level of the individual. The thesis of the modularity of emotion as it is usually understood can be seen as an attempt to reduce what happens at the level of the individual, the emotion, to what happens at the sub-personal level, the module. If there are affective modules similar to the biological modules described earlier, they will relate the sub-personal level with the collective level, bypassing in a sense the individual.

Are there any affective modules? I am not a biologist and unfortunately I do not have any object or exemplar that I could show and say "Here! Look. This is an affective module." Furthermore the level at which the function of affective modules should be defined often remains hidden from us. Affective coordination is such a usual thing that takes place all the time that we generally do not notice it. The existence of a particular mechanism whose task would be to fulfill this 
Biological Modules and Emotions

function seems superfluous. One of the reasons for this invisibility of affective coordination is that we cannot step out of it in order to observe it from the outside. Unlike cell differentiation or the formation of a border that separates cell populations in the case of affective coordination, we cannot directly observe the global effect, but only its repercussion at the individual level. In an attempt to turn this difficulty, I will introduce the topic of affective coordination by way of a fiction, more precisely of a "science-fiction," specifically, by way of an extract from a conference that will be given by Vulcan scientist Yram Rekab at the Federation's Star Academy on star date 29,036 and which I have been lucky enough to discover in an ancient chest abandoned in my cellar. Rekab, you will remember, is an anthropologist specializing in non-federation species and the topic of her conference that day was (will be) the origin of the Borg. ${ }^{6}$

\section{III. "Resistance Is Futile"}

There has been much speculation about the origin of the Borg. Where is it from? How did it begin? Clearly this is not a biological species, at least not today. On the contrary, as we all know from painful experience, the Borg assimilates by force as many biological species as it can and transforms their members into Borg, parts of itself, things that are half living and half machine. Those that cannot be assimilated are simply killed. The goal of the assimilation of diverse species seems to be to augment the pool of technological knowledge and genetic material that is at the disposal of the Borg, thereby adding endlessly to its store of competence, knowledge, and power. When assimilation takes place, the individual's self disappears and he or she becomes one with the collective. Simultaneously, all the knowledge possessed by that individual becomes available to the Borg. The Borg only has one mind

6 For those who are not familiar with it, the television series Star Trek takes place in the future when humankind has discovered the technology of efficient space travel and is now part of a multi-galactic political organization, the Federation (the good guys) in which many different species of intelligent beings participate, for example, Humans and Vulcans. Opposing the Federation are various other species and civilizations, for example, the Klingon and Romulan empires, but also the Borg, who, as the name suggests, are Cyborgs, half mechanical and half living creatures. 
that is shared by all and that is why it makes sense to say as we do 'the Borg' rather than 'Borgs' for there are not many of them but only one in spite of the fact that the Borg is not spatio-temporally continuous.

The thesis I want to defend is that the Borg is a religion, a religion that, like Christianity or Buddhism, has a universal vocation. The Borg does not reproduce; members of the collective have lost the necessary autonomy. The Borg 'converts' other people. On many planets of the Federation, religions exist that are religions of the denial of the self. The self is nothing, these religions argue. It is an illusion and the goal of spiritual exercises is to abolish the self and become one with the universal mind. The Borg can be seen as a material and technological realization of this spiritual endeavour. This is what the Borg does; it materially, technologically creates a universal mind, a mind that is shared by all and obliterates the self. Given this, it would therefore make absolutely no sense to ask this illusion, the self, whether or not it wants be assimilated. Individuals are simply errors that should be eradicated.

The greatest objection to this hypothesis, which it must be said is not entirely new, comes from those who claim that the self of members of the collective is not destroyed entirely. It is argued that, even though each one is connected to all others and shares their mind and knowledge, individual members of the collective remain to some extent as poles of initiative. That is to say, they respond to local situations and their success in dealing with them is due to the fact that, thanks to their interconnection, they have access to all the knowledge of the collective. Furthermore, it is argued that there is no technology known to us that could react in real time to so many local situations as those that confront the billions of members of the collective. This last argument, however, is moot, given that we have no idea how it is technically possible for all members of the collective to access at all times the complete stock of knowledge of the Borg. We are nonetheless certain that this happens.

What prompts me to believe that the self of organisms assimilated into the Borg is properly extinguished is that when an individual is transformed into a member of the collective it immediately loses the ability to express its emotions. It may be objected that this incapacity to express emotions is purely strategic and does not indicate that members of the collective do not feel anything. Their apparent indifference 
Biological Modules and Emotions

to fear and complete absence of pity, and the methodical and emotionless way in which they proceed to their end, without rage, anger, or doubt, makes them formidable adversaries, who, it seems, can never be deterred from their goals, but only destroyed. In other words, this apparent insensitivity, it is argued, is there mainly to instill terror in the hearts of the Borg's victims. Perhaps is it true that the Borg still feels, but this objection itself illustrates precisely the point I am trying to make. The objection rests on the fact that Humans, and yes, even Vulcans, immediately interpret the Borg's absence of affective expression as a sign of attitudes and dispositions that, if they are not emotions as such, are clearly related to them, for example, resoluteness, determination, cruelty, and indifference. The adjective "strategic" in the above objection assumes that we anticipate that the Borg's behaviour will be consistent with what is suggested by the signs that we recognize or that we assume to be there. This in turn implies, first, that we do not understand the Borg's lack of emotional expression as affective silence. We give it meaning in terms of emotions. However, this spontaneous projection informs us about the type of creatures that we are but tells us nothing about the Borg. For us, there is no behaviour that is without a certain affective quality, none to which we do not attribute an emotional dimension of some sort. The term "strategic" also implies that affective expression is directly related to behaviour. The idea of strategy as it is used in the objection supposes that the lack of affect expressed by the Borg is unmistakenly associated by us with definite behaviours. Finally it suggests that the impassivity of the Borg will spontaneously orient us towards certain affective dispositions, such as fear, doubt, irresolution, and perhaps terror and confusion, as if the insensitivity of Borg itself were an action that had direct consequences on our own behaviour.

It therefore does not really matter whether or not members of the collective feel anything, for we spontaneously interpret their expressive passivity as revealing definite affective dispositions. What does matter for the argument concerning the disappearance of the self, however, is that (it) they do(es) not express anything. When we respond affectively as we do to the Borg's indifference, through anger, fear, repulsion, or disgust, we attempt to coordinate our actions to theirs. Unfortunately, this spontaneous effort of ours is doomed to failure because they cannot answer our affect. Unlike the action of their 
imagined insensitivity upon us, our emotional expression has no hold upon them. How is this failure visible? What demonstrates it is that we cannot individualize members of the collective. I do not mean by this that we cannot recognize that this "thing," half human and half machine, that is now coming towards us was not Borg half an hour ago but a data analyst working in engineering. It is, on the contrary, easy to recognize that members of the collective once were distinct individuals belonging to different species. However, what we cannot do is individualize them in action so to speak. There is nothing we can do that can evoke from a member of the collective a response that is not dictated by the collective.

Members of the collective do not react to affective expression because they do not need to. The Borg's mind is conscious of itself and the access of all to the complete store of information it contains is immediate and total. No individual needs therefore to coordinate his or her actions to those of another precisely because they are not individuals but part of a whole. The smooth functioning of the various parts of the Borg is centrally directed. There is no need for local and individual coordination in this situation because no one is uncertain about the intentions of another towards him. That is why members of the collective neither express nor recognize emotions. They have no use for that device.

\section{A Coordination Module}

The manuscript is unfortunately incomplete. The Borg, unlike us, does not need to express or recognize emotions because what are called in the fiction "members of the collective" are actually its parts. They have no autonomy. Between them, there may be some room for play as in a mechanism, but none of them is a pole of initiative. We, on the contrary, are a highly social species. This means that for each one of us most of the advantages and disadvantages that we can receive in life come from other members of our own species. Each individual is a pole of initiative. Everyone can initiate a chain of action that is unpredictable to others and yet can have fundamental consequences upon them. Because of this, knowledge of the intentions and dispositions of each individual toward others is of paramount importance. Conflict, cooperation, reproduction, alliances, and sustained relations 
Biological Modules and Emotions

all depend to some extent on the intentions others harbour towards us and on how they understand our dispositions towards them. Given this, it would seem like a good idea if members of a species similar to ours had a way of informing one another of their mutual intentions and dispositions. This would allow them to coordinate their action and interact more frequently in ways that are mutually beneficial.

Since Adam Smith (1759) ${ }^{7}$ and Francis Hutcheson (1726), emotions, or at least some emotions, have been viewed as playing precisely this role. Most authors, however, including modern ones like Robert Frank (1988), Alan Gibbard (1990), or Peter Strawson (1963), have usually focused on the inner sentiment rather than on the expression of affect itself. This approach leads to two important difficulties. The first, which has been extensively studied, can be called the problem of sincerity detection. ${ }^{8}$ A device that allows one organism to inform another of his or her disposition and intentions towards that second organism will play its role only if it transmits reliable information, that is to say, if cheaters can be detected and truthful messages regularly recognized. The signal must be consistent with the inner sentiment, and the problem of sincerity detection is that of recognizing when that condition is satisfied and when it is not.

The second difficulty, has received a lot less attention but is perhaps even more important and can be called the problem of indeterminacy. As de Waal and Aureli wrote: "if two individuals compete over a particular resource, they need to take into account not only the value of the resource and the risk of bodily harm, but also the value of their relationship. Sometimes the resource may not be worth straining a cooperative relationship" (de Waal and Aureli 1999, 122). However, it is clear that for social animals that are sophisticated enough to distinguish between the partners with whom they interact the value of a given relationship for one individual is not independent of the value of that same relationship for the other individual involved. The value of a relationship is related to reiterated interaction, and how an organism behaves in a specific situation is indicative of the importance it

7 Adam Smith The Theory of Moral Sentiments (1759) (Indianapolis: Liberty Classic, 1976).

8 See, for example, Skyrms (1996), esp. chap. 5, and Frank (2004). 
gives to future interaction. It is a conceptual truth therefore that the value further interaction with another organism has for one organism is not independent of the value it has for the other organism. Thus, the value my relationship with you has for me is not independent of the value your relationship with me has for you, and vice versa. More generally, my intentions towards you are not independent of your intentions towards me. They will change depending on how yours turn out to be. Given that this is true of all of us, there is a sense in which our intentions towards one another are radically indeterminate. But if this is the case, how is it possible for me to inform you of my intention towards you if it is not already determined but awaits information concerning your intention towards me in order to reach a definite form? And how is it possible for you to inform me since you are in exactly the same situation?

If this description of the situation is accurate, then there is no system of communication, classically understood as an exchange of messages carrying information, which can resolve these two difficulties. However, there is another way to achieve the desired goal of coordination and mutually beneficial interactions while avoiding these two pitfalls. It is to have the intentions of every individual partially determined by other individuals. Organisms could thus coordinate by converging towards complementary intentions and actions. From this point of view, the expression of emotion should not be seen as a system of signalling but as a way in which one organism acts upon the intentions and dispositions of another. Affective expression is a mechanism through which individuals reciprocally determine one another's mutual intentions. This interaction is not an exchange of messages or information; it contains no representation but is a form of reciprocal action of one organism upon another. This may seem strange at first, but when biologists talk about animal communication this is often pretty much what they describe, even if they do not use exactly this language. This is what bird songs and mating rituals are about; the vocabulary of messages and information is purely metaphorical in this context. ${ }^{9}$ Furthermore, there is beginning to be quite a bit of neurological evidence that, through reciprocal expression of

9 See, for example, Hauser and Konishi (2003), 701. 
Biological Modules and Emotions

affect, mammals, and primates in particular, do things to one another. When one organism perceives the expressed emotion of another, that perception modifies it own inner state (Perret 2003). It triggers the production of a hormone or neurotransmitter, or initiates a neurological or physiological reaction that is immediate and automatic. Actually, we also know that certain affective displays are necessary for the normal development of various social skills and abilities and even some neurological components (Levine et al. 1999; Wallen and Tannenbaum 1999; Perret 2003; Cheney and Seyfarth 1999; Gergely at al. 2002; Decety 2002).

The first advantage of looking at the expression of affect in this way is that the problem of indeterminacy is solved. Determinate intentions do not precede the dynamic of expression but appear at the end of the process. There is no need to presuppose them. The goal of the interaction is to determine them. How is this done? A good example of this is the regulation of voice pitch during conversation. Empirical studies have shown that during conversation the voice pitch of each speaker is closely related to that of his or her partners. The pitch of each speaker varies in response to the modulation of the pitch of others, and this close coordination is strongly related to the emotional tone of a conversation (Cowley 1997; 1998). In this way, the pitch of one speaker acts upon the pitch of another but also upon the emotional state or disposition of the other. These changes in voice pitch and tone are part of what makes a conversation progress towards anger, distress, or laughter. They do not follow but precede this progression. The process is generally wholly unconscious. Most of the time we are not aware of it and notice our reciprocal vocal adaptation only when emotions erupt on the front stage and we start shouting insults at one another. However, from the point of view of the coordination of voice pitch, there is no discontinuity between the moment when speakers would describe the situation in terms of emotions and the part of the conversation that came before. The process takes place at a sub-personal level; the coordination is taken care of by neurologically closely integrated devices of perception and expression of affect (Adolphs 2003; Perret 2003), but its consequence appears at the level of social coordination of two or more individuals.

The second advantage of looking at the expression of affect in this way is that the problem of sincerity detection is radically transformed. 
If we do not start out from determined intentions but only reach them through a process of dynamic interaction in which each individual partially determines the intentions of others, then the question of the sincerity of affective expression is no longer one of knowing if an agent is cheating, in the sense of discovering whether he or she is sending out a message that is inconsistent with his or her real intentions. As Don Ross and I have argued, in this new context, the question of sincerity is not one of distinguishing truthful messages from unreliable ones but of holding agents to their commitments (Ross and Dumouchel 2004a, 2004b). This is done in two ways. First, important coordination equilibria result in strong affects. Robert Frank believes that strong affects guarantee the truth of certain messages, especially if the expression of such affect is to a large extent involuntary (Frank 1988). The reason for this is that Frank rightly assumes that strong affects increase commitment. Given this, if the coordination of intention through affective expression is associated with strong affects, then the probability that agents will be committed to that solution of coordination will also augment. The second way in which agents are held to their commitments is that standard expressions of emotions, which are the end results of the dynamic of coordination and are associated with definite intentions, have evolved as convention of coordination. ${ }^{10}$ It is by definition to the advantage of everyone to respect conventions and those who transgress them are ostracized. In other words, agents commit themselves through the fact that they express their emotions in standardized ways that everyone associates with definite intentions. They make in short public commitments. Of course, agents can cheat and they sometimes do. They do not always live up to their affective commitments, but the fact that more or less seven standard emotional displays are universally recognizable suggests that these conventions are generally respected.

In what way can this coordination be viewed as resting on modules similar to those that were described earlier in the second section of this text? At this point the answer to this question can only be highly speculative. Nonetheless, in closing, consider the following points. First,

10 This is a very natural way of understanding Paul Ekman's results concerning the expression of emotion. What we recognize as basic emotions are fixed points of affective coordination. 
Biological Modules and Emotions

it seems possible to argue that (at least some of) the devices responsible for affective coordination are autonomous in the required sense. Once initiated affective coordination follows its course independently of many other events that take place in the environment. The process is to some extent self-sustaining. Affective expression from one organism triggers a similar type of response from another organism that in turn acts upon the first until an equilibrium of coordination is found. The self-organizing aspects of affective coordination suggest that the underlying mechanisms remain stable under a wide range of environmental changes. Second, it seems that they also satisfy the requirement of reiterated use in different domains, for example, perception and action in both the visual and the audible domains. In fact it is precisely that plasticity which creates problems for our concepts of emotions. Because of that, it is only under a sufficiently abstract description that the module can be defined as the same, only in the sense that the description of its function is coordination among co-specifics. ${ }^{11}$ The module of affective expression and perception can be applied to any situation that can be described as one where there is a need for coordination between different organisms. Thirdly, the modules are also hierarchically organized, since the expression of many emotions such as anger, fear, and disgust depend on neural systems that also seem to function as modules. Fourthly, many of these systems are relatively ancient and seem to have been doing the same thing, for example, fear conditioning, in many different species that are sometimes phylogenetically quite distant (LeDoux 1998; Adolphs 2003). Finally, just as in the case of many biological modules, the consequences or function of these modules, social coordination, takes place at the level of a group of organism while the module is described at a sub-personal level.

\section{References}

Adolphs, R. 2003. Neural systems for recognizing emotions in humans. In The Design of Animal Communication, ed. M. D. Hauser and M. Konishi, 187-211. Cambridge (Mass.): MIT Press.

11 Mostly but not exclusively since it seems clear that we can to some extent reach affective coordination with some other mammals like dogs or horses. 
Paul Dumouchel

Ameisen, J.-C. 1999. La Sculpture du vivant. Le suicide cellulaire ou la mort créatrice. Paris: Seuil.

Borycki, A.-G. 2003. Sonic hedgehog and wnt signaling pathways during development and evolution. In Modularity in Development and Evolution, ed. G. Schlosser and G. Wagner. Chicago: Chicago University Press.

Camazine, S., J.-L. Deneubourg, N. R. Franks, J. Sneyd, G. Theraulaz, and E. Bonabeau. 2003. Self-Organization in Biological Systems. Princeton: Princeton University Press.

Cheney, D. L., and R. M. Seyfarth. 1999 Mechanism underlying the vocalizations of nonhuman primates. In The Design of Animal Communication, ed. M. D. Hauser and M. Konishi, 627-43. Cambridge (Mass.): MIT Press.

Cosmides, L., and J. Tooby. 2000. Evolutionary psychology and the emotions. In Handbook of Emotions, ed. M. Lewis and J. Haviland-Jones, 91-115. New York: The Guilford Press.

Cowley, S. J. 1997. Of representations and language. Language and Communication 17(4): 279-300.

Cowley, S. J. 1998. On timing, turn-taking, and conversation. Journal of Psycholinguistic Research 27(5): 541-71.

de Celis, J. F. 2003. The notch signaling module. In Modularity in Development and Evolution, ed. G. Schlosser and G. Wagner. Chicago: Chicago University Press.

de Waal, F.B.M., and F. Aureli. 1999. Conflict resolution and distress alleviation in monkeys and apes. In The Integrative Neurobiology of Affiliation, ed. S. Carter, I. Lederhendler, and B. Kirkpatrick. Cambridge (Mass.): MIT Press.

Decety, J. 2002. Neurobiologie des représentations motrices partagées. In Imiter pour découvrir l'humain, ed. J. Nadel and J. Decety, 105-30. Paris: PUF.

Fodor, J. 1983. The Modularity of Mind. Cambridge (Mass.): MIT Press.

Frank, R. 1988. Passions within Reason: The Strategic Role of the Emotions. New York: Norton.

Frank, R. 2004. In defense of sincerity detection: Response to Ross and Dumouchel. Rationality and Society 16(3): 287-305.

Gergely, G., O. Koos, and J. S. Watson. 2002. Perception causale et rôle des comportements immitatifs des parents dans le développement socioémotionnel précoce. In Imiter pour découvrir l'humain, ed. J. Nadel and J. Decety, 59-82. Paris: PUF. 
Gibbard, A. 1990. Wise Choices, Apt Feelings: A Theory of Normative Judgment. Oxford: Oxford University Press.

Hauser, M. D., and M. Konishi. 2003. The Design of Animal Communication. Cambridge (Mass.): MIT Press.

Hobbes, T. 1651. Leviathan. Indianapolis: Hackett.

Hutcheson, F. [1726] 2004. An Inquiry into the Original of Our Ideas of Beauty and Virtue. Wolfgang Leidhold, ed. Indianapolis: Liberty Classic.

Kardon, G., T. A. Heanue, and C. T. Tabin. 2003. The Pax/Six/Eya/Dach network in development and evolution. In Modularity in Development and Evolution, ed. G. Schlosser and G. Wagner. Chicago: University of Chicago Press.

Kelley, E. F. 2002. Making Sense of Life. Cambridge (Mass.): Harvard University Press.

LeDoux, J. 1998. The Emotional Brain. New York: Simon \& Schuster.

Levine, S., D. M. Lyons, and F. Schatzberg. 1999. Psychobiological consequences of social relationships. In The Integrative Neurobiology of Affiliation, ed. S. Carter, I. Lederhendler, and B. Kirkpatrick, 83-89. Cambridge (Mass.): MIT Press.

Perret, D. I. 2003. A cellular basis for reading minds from faces and actions. In The Design of Animal Communication, ed. M. D. Hauser and M. Konishi, 159-85. Cambridge (Mass.): MIT Press.

Ross, D., and P. Dumouchel. 2004a. Emotions as strategic signals. Rationality and Society 16(3): 251-86.

Ross, D., and P. Dumouchel. 2004b. Sincerity is just consistency: Reply to Frank. Rationality and Society 16(3): 207-318.

Schlosser, G. 2003. The role of module in development and evolution. In Modularity in Development and Evolution, ed. G. Schlosser and G. Wagner, 525. Chicago: Chicago University Press.

Skyrms, B. 1996. Evolution of the Social Contract. Cambridge: Cambridge University Press.

Sloman, A. 2002. How many separately evolved emotional beasties live within us? In Emotions in Humans and Aritifacts, ed. R. Trappl, P. Petta, and S. Payr, 35-114. Cambridge (Mass.): MIT Press.

Smith, A. [1759] 1976. The Theory of Moral Sentiments. Indianapolis: Liberty Classic.

Strähle, U., and P. Blader. 2003. The basic helix-loop-helix proteins in vertebrate and invertebrate neurogenesis. In Modularity in Development and 
Paul Dumouchel

Evolution, ed. G. Schlosser and G. Wagner. Chicago: Chicago University Press.

Strawson, P. 1963. Freedom and resentment. Proceedings of the British Academy 48: 1-25.

von Dassow, G., and E. Meir. 2003. Exploring modularity with dynamical models of gene networks. In Modularity in Development and Evolution, ed. G. Schlosser and G. Wagner. Chicago: Chicago University Press.

Wallen, K., and P. L. Tannenbaum. 1999. Hormone Modulation of sexual behavior and affiliation in rhesus monkeys. In The Integrative Neurobiology of Affiliation, ed. S. Carter, I. Lederhendler, and B. Kirkpatrick, 101-18. Cambridge (Mass.): MIT Press. 